\title{
Preface: Special issue dedicated to the tenth international conference on optimization techniques and applications (ICOTA 10)
}

\author{
Panos M. Pardalos ${ }^{1} \cdot$ Kok Lay Teo $^{2,3} \cdot$ Rentsen Enkhbat $^{4}$
}

Received: 30 January 2019 / Accepted: 20 February 2019 / Published online: 11 March 2019

(c) Springer-Verlag GmbH Germany, part of Springer Nature 2019

\begin{abstract}
This special issue of the optimization letters contains, after stringent reviewing process, 12 papers from among many submissions received from the participants of the tenth international conference on optimization techniques and applications, which was held in Ulaanbaatar, Mongolia during 23-26 July, 2016. The topics covered by these 12 papers include: optimal control, global optimization, optimization techniques and their applications.
\end{abstract}

This special issue of the Optimization Letters is dedicated to the tenth international conference on optimization techniques and applications, which was held in Ulaanbaatar, Mongolia during 23-26 July, 2016. Many submissions were received from the conference participants. After stringent reviewing process, 12 papers are accepted for inclusion in this special issue.

The ICOTA conference series is an official conference series of Pacific Optimization Research Activity Group, called POP. The 10th ICOTA conference was supported by the National University of Mongolia, University of Humanities, German-Mongolian

Kok Lay Teo

K.L.Teo@curtin.edu.au

Panos M. Pardalos

pardalos@ufl.edu

Rentsen Enkhbat

renkhbat46@yahoo.com

1 Department of Industrial and Systems Engineering, University of Florida, P.O. Box 116595, Gainesville, FL 32611-6595, USA

2 Department of Mathematics and Statistics, Curtin University, Kent Street, Bentley, Western Australia 6102, Australia

3 Coordinated Innovation Center for Computable Modeling in Management Science, Tianjin University of Finance and Economics, Tianjin 300222, China

4 National University of Mongolia, Street Sukhbaatar, P.O. Box 46/635, Ulaanbaatar 14201, Mongolia 
Institute for Resources and Technology, the Commission for Developing Countries of the International Mathematical Union, and the Abdus Salam International Centre for Theoretical Physics. Like previous ICOTA conferences, the 10th ICOTA conference provided an international forum for researchers and practitioners to exchange recent findings and developments in theory, methods and applications in optimization and control, and to identify new research challenges as well as promising research developments on all issues and topics related to optimization and control and their applications.

The papers included in this special issue are: An Li, "Optimal control of implicit control systems and its application to differential complementarity problems"; Alexander S. Strekalovsky, "Global Optimality Conditions and Exact Penalization"; Hong Seng Sim, Wah June Leong, and Chuei Yee Chen, "Gradient Method with Multiple Damping for Large-scale Unconstrained Optimization"; Anatoly Antipin and Elena Khoroshilova, "Controlled dynamic model with boundary-value problem of minimizing a sensitivity function"; S. Batbileg, N. Tungalag, A. Anikin, A. Gornov and E. Finkelstein, "A Global Optimization Algorithm for Solving Four-Person Game"; Huifang Wang, Lingchen Kong and Jiyuan Tao, "The linearized alternating direction method of multipliers for sparse group LAD model"; Yanjun Jiang, Dachuan Xu, Donglei Du, and Dongmei Zhang, "An Approximation Algorithm for the Dynamic Facility Location Problem with Outliers"; Lei Wang, Jinlong Yuan, Changzhi $\mathrm{Wu}$, and Xiangyu Wang, "Practical algorithm for stochastic optimal control problem about microbial fermentation in batch culture"; Enkhbat Rentsen and Enkhbayar Jamsranjav, "A Note on Maxmin Problem”; Yue Shi, Zhiguo Feng, and Ka Fai Cedric Yiu, "A Descent Method for Least Absolute Deviation Lasso Problems"; Lu Han, Dachuan Xu, Donglei Du and Chenchen Wu, "A 5-approximation algorithm for the k-prize-collecting Steiner tree problem".

We, as guest editors, would like to thank all authors who showed interest in this special issue and all the reviewers who accepted and provided their expert and constructive comments. Our thanks also go to the National University of Mongolia, University of Humanities and German-Mongolian Institute for Resources and Technology, the Commission for Developing Countries of the International Mathematical Union and the Abdus Salam International Centre for Theoretical Physics for their support. Thanks to the Editors-in-Chief of Optimization Letters, P. Krokhmal and O.A. Prokopyev, for their interest and continuous support. We hope that the reader will find the contributions of this special issue enjoyable and inspiring.

\section{Guest Editors:}

Panos Pardalos, Kok Lay Teo and Rentsen Enkhbat January 2019

Publisher's Note Springer Nature remains neutral with regard to jurisdictional claims in published maps and institutional affiliations. 